\title{
Electron Optical Requirements for Sub-Nanosecond Microscopy
}

\author{
Bryan W. Reed, Michael Armstrong, and Wayne E. King
}

Chemistry and Materials Science Directorate, University of California, Lawrence Livermore National Laboratory, Livermore, CA 94550

It is self-evident that improving the time resolution of an in-situ technique opens up entirely new realms of physical processes for investigation. The field of optics, with its well-developed femtosecond techniques, has been reaping these benefits for some time. Short-pulse x-ray and ultrafast electron diffraction (UED) approaches are very much on the rise and are producing stunning results of their own.

A movement is afoot to similarly improve the time resolution of imaging in the transmission electron microscope (TEM). The TEM has a unique ability to produce high-quality real-space images with sub-nanometer resolution and high frame rates. Most current in-situ TEM investigations are limited to video-rate imaging, but this is more a limitation of the detector than of the electron optics. Previous work at TU Berlin has demonstrated the ability of the TEM to take images and movies on a nanosecond time scale [1]. This has formed the basis of efforts at Lawrence Livermore National Laboratory and elsewhere to push these techniques to their limits, developing the next generation of so-called dynamic TEM's (DTEM's), which are intended to achieve sub-nanosecond time resolution.

As part of this effort, we have investigated the theoretical limits of ultrafast electron microscopy. We have found that the design of the entire column may have to be re-thought in order to operate well under the high beam currents (several $\mathrm{mA}$ ) occurring in a DTEM pulse. The gun (replaced with a pulsed photocathode) has to be optimized not for stability and spatial coherence (as in standard TEM) but for raw electron output (Figure 1). This suggests some significant redesigns. Boersch, space charge, and trajectory displacement effects, while relatively minor nuisances in standard TEM, become stringent limits on the DTEM capabilities, while the effects that normally limit spatial resolution (an optimal compromise between aberrations, defocus, and diffraction limits) are almost irrelevant (Figure 2). In fact, the current DTEM resolution limit is probably not in the objective lens at all. The intermediate/projector lens system will encounter the same trajectory displacement problems that limit resolution in electron projection lithography systems to some tens of nm [2].

Having identified the limitations in present systems, we can develop approaches for surpassing them. An important step will be to incorporate the results in related fields (accelerator physics, projection lithography, and streak camera design) to help us fundamentally rethink how a fast TEM should work. Future systems may be built around radio-frequency $\mathrm{MeV}$ electron sources, with pulse compression, dynamic aberration correction, advanced detection methods, and unusual lens designs.

\section{References}

[1] H. Domer and O. Bostanjoglo, Rev. Sci. Inst. 74 (2003) 4369.

[2] G. H. Jansen, Coulomb Interactions in Particle Beams, Academic, San Diego, 1990.

[3] P. Stadelmann, Java EMS, http://cimewww.epfl.ch/people/stadelmann/jemswebsite/jems.html 
[4] This work was performed under the auspices of the U.S. Department of Energy by the University of California, Lawrence Livermore National Laboratory under contract No. W-7405-Eng-48.

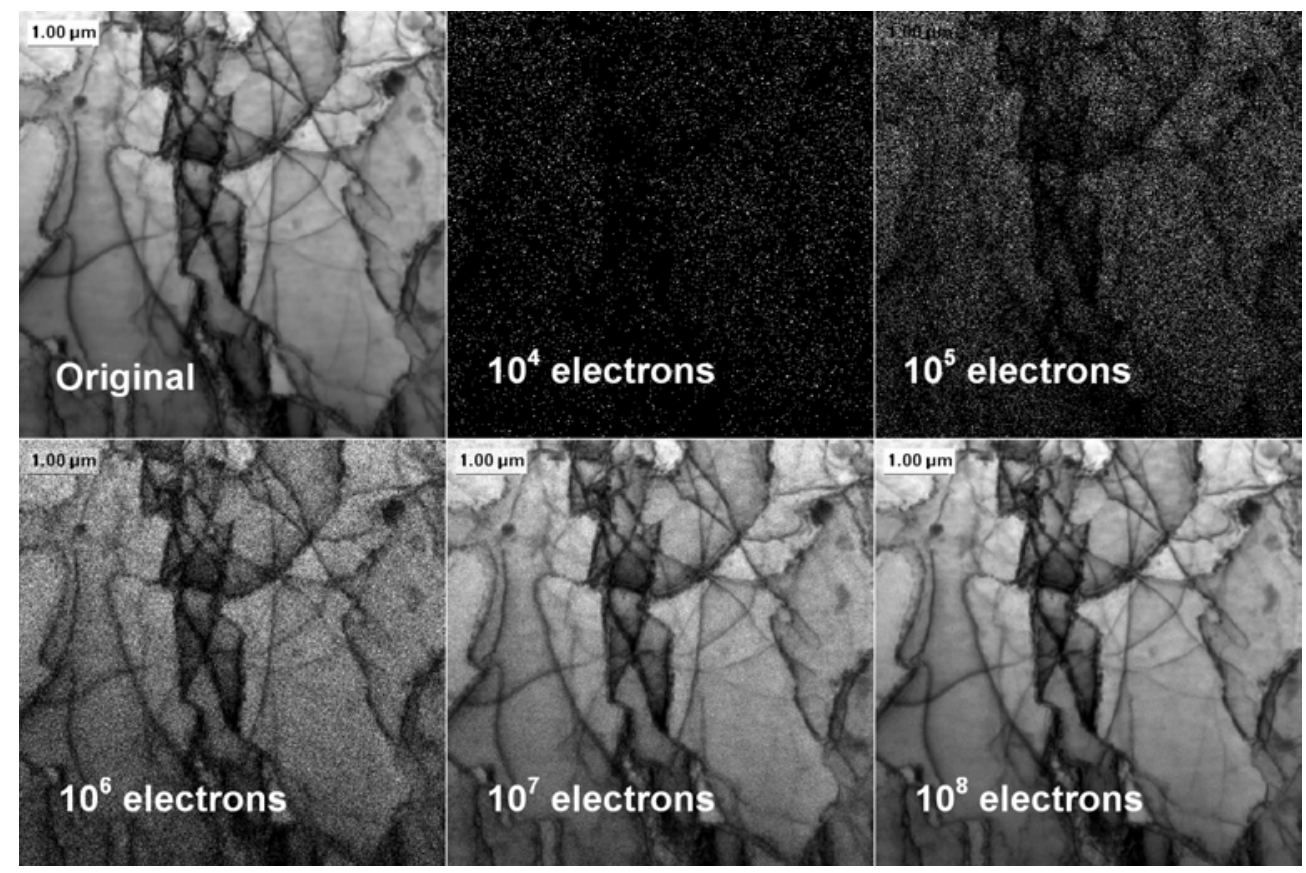

Figure 1. Simulation of the contrast possible in a 512 x 512 pixel image with varying numbers of electrons. $10^{6}-10^{8}$ electrons are required for a good image. With a $1 \mu \mathrm{m}$ illumination area, $10 \mathrm{mrad}$ convergence angle, and $1 \mathrm{~ns}$ exposure time, this implies a source brightness of order $10^{7}-10^{9} \mathrm{~A} \mathrm{~cm}^{-2}$ steradian $^{-1}$ and a current of 0.16-16 mA at the cathode. These are very challenging numbers for a steady-state conventional TEM gun, but are achievable by photocathodes.

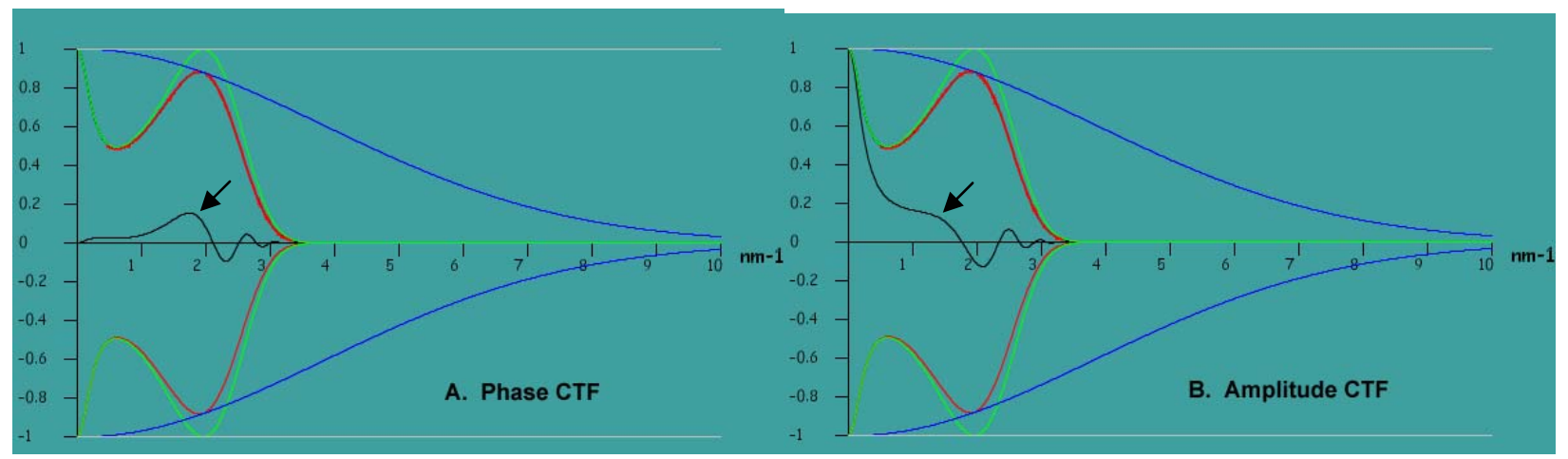

Figure 2. Phase and amplitude contrast transfer functions (black curves with arrows) for typical parameters that may be encountered in a DTEM, calculated by Java EMS [3]. E $=100 \mathrm{keV}, \Delta \mathrm{E}=5$ $\mathrm{eV}, \mathrm{C}_{\mathrm{S}}=\mathrm{C}_{\mathrm{C}}=2 \mathrm{~mm}$, convergence semi-angle $10 \mathrm{mrad}$, defocus $105 \mathrm{~nm}$, no objective aperture. Green $=$ spatial coherence envelope, blue $=$ temporal coherence envelope, red $=$ product of envelopes. Sub-nanometer resolution is predicted (particularly with amplitude contrast mechanisms) if objective lens aberrations dominate the resolution limit. The experimental limit is closer to 100 $\mathrm{nm}$ [1], and is likely due to a combination of low signal levels and electron-electron scattering in the post-sample crossovers. 